(7) $f=g \circ(\varphi \mid A \cup B)$,

(8) $g \mid \varphi(A)$ and $g \mid \varphi(B)$ are continuous.

Thus, $g$ is continuous and hence it is extended to a continuous map $h: S \rightarrow \bar{X}$. Then $h \circ \varphi: X \rightarrow \bar{Y}$ is an extension of $f$.

Theorem 11 does not hold in general if we drop the assumption that $A$ and $B$ are zero-sets of $X$.

EXAMPLE. Let us put

$$
\begin{aligned}
& X=W\left(\omega_{1}+1\right) \times W\left(\omega_{1}+1\right)-\left(\omega_{1}, \omega_{1}\right), \\
& A=W\left(\omega_{1}\right) \times \omega_{1}, \quad B=\omega_{1} \times W\left(\omega_{1}\right) .
\end{aligned}
$$

Then $A$ and $B$ are $C$-embedded in $X$ but $A \cup B$ is not.

Added in proof. In case $Y=[0,1]$ or $(0,1)$ Theorem 7 has been proved, also by M. Starbird (The normality of products with a compact or a metric factor, $\mathrm{Ph} . \mathrm{D}$. Thesis Univ. of Wisconsin, 1974).

The converse of Theorem 5 holds. Cf. K. Morita and T. Hoshina, O-embedding and the homotopy extension property, to appear in Gen. Topology and Appl.

\title{
References
}

11] R. Arens, Extension of coverings, of pseudometrics, and of linear-space-valued mappings, Can. J. Math. 5 (1953), pp. 211-215.

[2] K. Borsuk, Theory of Retracts, Warszawa 1967.

[3] C. H. Dowker, On a theorem of Hanner, Ark. f. Mat. 2 (1952), pp. 307-313.

[4] - Homotopy extension theorems, Proc. London Math. Soc. 6 (3) (1956), pp. 100-116.

[5] S. T. Hu, Theory of Retracts, Detroit 1965.

[6] K. Morita, Topological completions and $M$-spaces, Sci. Rep. Tokyo Kyoiku Daigaku, Sect. A 10 (1970), pp. 271-288.

[7] - Čech cohomology and covering dimension for topological spaces, Fund. Math. 87 (1975), pp. 31-52.

[8] M. E. Rudin, $A$ normal space $X$ for which $X \times I$ is not normal, Fund. Math. 73 (1971), pp. 179-186.

[9] H. L. Shapiro, Extensions of pseudometrics, Can. J. Math. 18 (1966), pp. 981-998.

Accepté par la Rédaction le 8. 11. 1973

\section{Conservative extensions and the two cardinal theorem for stable theories}

by

John T. Baldwin (Chicago, IIl.)

Abstract. Definimion. $\mathcal{B}$ is a conservative extension of $\mathcal{A}$ if $\mathcal{B} \zeta \mathbb{A}$ and if for every set $X$ definable in $\Re X \cap|\mathcal{A}|$ is definable in $A$. We use this definition to give a short proof of Lachlan's result: If $\mathcal{A}$ is a proper elementary submodel of $\mathcal{B}$ and $\mathcal{A}, \mathcal{B}$ are models of a stable theory $T$ but $D(\mathcal{A})=D(\Re)$ then there exists a proper elementary extension $\mathcal{C}$ of $\Re$ with $D(\mathcal{C})=D(\mathscr{A})$.

Let $L$ be a countable first order language and $D$ a unary $L$-formula. In [3] Lachlan proved that if $T$ is stable, $A$ and $\mathcal{B}$ are models of $T$ with $|\mathfrak{A}| \neq|\mathfrak{B}|, \mathfrak{A} \leq \mathfrak{B}$ and $D(\mathscr{A})=D(\Re)$ then there exists a proper elementary extension $\mathcal{C}$ of $\mathscr{B}$ with $D(\mathcal{C})=D(\mathfrak{t})$. We give a simpler proof of this result. In addition, if $\mathcal{B}$ is countable we can weaken the hypothesis " $T$ is stable" to " $\mathfrak{B}$ is a conservative extension of $\mathfrak{A}$ ". We define the notion of conservative extension below and remark that $T$ is stable if and only if every elementary extension of every model of $T$ is conservative. Our proof of the main theorem is evidence for taking this as the definition of a stable theory.

In general our notation follows [9]. We vary however by letting $F_{n}(L)$ denote the set of $L$-formulas with $n$ free variables and $F_{n}(X)$ the formulas with $n$ free variables in the expansion of $L$ which names each member of $X$. We write $|\mathfrak{A}|$ for the universe of the structure $A$ and || for cardinality. Thus $\| A A$ denotes the cardinality of the universe of $\mathcal{A}$. If $\mathcal{A}$ is an $L$-structure and $X \subseteq \mathcal{A}, A \in F_{n}(X)$ then

$$
A(\mathcal{A})=\left\{\left\langle a_{0}, \ldots, a_{n-1}\right\rangle \mid \mathcal{A} \vDash A\left(a_{0}, \ldots, a_{n-1}\right)\right\} .
$$

We particularly want to acknowledge our debt to Andreas Blass for many stimulating conversations on the notion of conservative extension and for suggesting some simplifications in the proofs here.

The following definition came to our attention because of its application to Peano arithmetic in $[6,7]$.

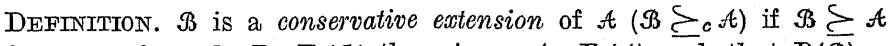
and for every formula $B \in F_{n}(\Re)$ there is an $A \in F_{n}(\mathcal{A})$ such that $B(\bar{\beta}) \cap$ $\cap|\mathfrak{A}|^{n}=A(\mathcal{A})$. 
For the definition of a stable theory see [8]. The sufficiency in the following theorem is a special case of [8, Theorem 3.1]. A proof without recourse to rank is announced in [2] and appears in [1]. The necessity is an easy application of the compactness theorem.

THEOREM 1. A complete first order theory $T$ is stable if and only if every elementary extension of every model of $T$ is conservative.

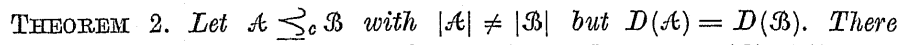
exists an elementary extension $\mathcal{C}$ of $\mathcal{B}$ and an element $o \in|\mathcal{C}|-|\mathcal{B}|$ such that each formula $B \in \mathbb{F}_{2}(\Re)$ either $\mathrm{C} \vDash \nabla v_{0}\left(B\left(c, v_{0}\right) \rightarrow \neg D\left(v_{0}\right)\right)$ or $\mathrm{C} \vDash B(c, a)$ for some $a \in D(\mathfrak{t})$.

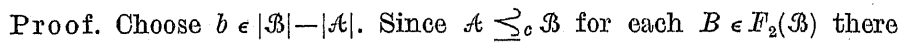
exists $B^{\prime} \in F_{2}(\mathfrak{A})$ with $B(\mathfrak{B}) \cap|\mathcal{A}|=B^{\prime}(\mathfrak{A})$. Let $\Gamma$ be the following set of sentences in $L$ enriched by names for the members of $\mathcal{B}$ and a new constant $e$.

$$
\begin{aligned}
\operatorname{Th}(\Re,|\Re|) \cup\{c \neq b|b \in| \Re \mid\} & \cup \\
\cup\left\{\neg \mathbb{H} v_{0} B\left(c, v_{0}\right) \wedge D\left(v_{0}\right) \mid \Re \vDash \neg \mathbb{H} v_{0} B^{\prime}\left(b, v_{0}\right) \wedge D\left(v_{0}\right)\right\} & \cup \\
& \cup\left\{B(c, a) \mid \Re \vDash B^{\prime}(b, a) \text { and } a \in D(\mathfrak{H})\right\} .
\end{aligned}
$$

It suffices to show that $\Gamma$ is consistent. For this, let $F_{0}, \ldots, F_{k}, G_{0}, \ldots, G_{l}$ $\in F_{2}(\Re)$ be such that $\urcorner \mathbb{H} v_{0} F_{i}\left(C, v_{0}\right) \wedge D\left(v_{0}\right) \in \Gamma$ for $i \leqslant k$ and $G_{j}\left(c, a_{j}\right) \in \Gamma$ for $j \leqslant l$. (The same $G_{j}$ may occur with different $a_{j}$ 's.) The result follows if $H(\mathscr{B})$ is infinite where

$$
H\left(v_{0}\right)=\bigwedge_{0}^{k} \neg \mathbb{H} v_{1}\left(F_{i}\left(v_{0}, v_{1}\right) \wedge D\left(v_{1}\right)\right) \wedge \bigwedge_{0}^{l} G_{j}\left(v_{0}, a_{j}\right) .
$$

But since $D(\mathfrak{A})=D(\Re)$, letting

$$
H^{\prime}\left(v_{0}\right)=\bigwedge_{0}^{k} \neg \mathbb{H} v_{1}\left(F_{i}^{\prime}\left(v_{0}, v_{1}\right) \wedge D\left(v_{1}\right)\right) \wedge \bigwedge_{0}^{l} G_{j}^{\prime}\left(v_{0}, a_{j}\right),
$$

$H(\mathscr{B}) \supset H^{\prime}(\mathfrak{S})$. Since $H^{\prime} \in H_{1}(\mathfrak{A})$ and $\mathscr{B} \vDash H^{\prime}(b)$ with $b \in|\mathfrak{B}|-|\mathfrak{A}| ; H^{\prime}(\mathfrak{A})$ and hence $H^{\prime}(\mathfrak{B})$ is infinite.

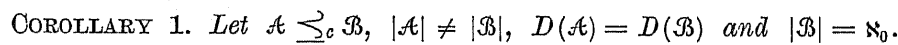
Then there exists a proper elementary extension $\mathrm{C}$ of $\mathcal{B}$ with $D(\mathcal{C})=D(\mathfrak{A t})$.

Proof. Define $\Gamma$ as in the proof of theorem. By the omitting types theorem (see especially the formulation in 4) there is a model of $T$ omitting the type $\left\langle D\left(v_{0}\right), v_{0} \neq a\right\rangle_{a \in D(f)}$.

For the general case $\left(|B|>\aleph_{0}^{\prime}\right)$ we finish the proof exactly as Lachlan did. The following question will probably require some additional । techniques.

Let $A \coprod_{c} B, D(A)=D(B),|A| \neq|B|$. Does there exist $O$ such that $B \coprod_{c} C$ and $D(A)=D(B)=D(C)$ ?
We have learned that the innovation of Theorem 2 was discovered independently but earlier by M. Daniel Lascar, Types definissible et produit de types, C. R. Acad. Sci. Paris, t. 276 (9 Mai 1973).

\section{References}

[1] J. T. Baldwin, Countable theories categorical in uncountable power, $\mathrm{Ph} . \mathrm{D}$. Thesis, Simon Fraser University, 1971.

[2] - A note on definability in totally transciendental theories, Notices of A.M.S. 70T-E87, 17 (1970), p. 1073.

[3] A. H. Lachlan, A property of stable theories, Fund. Math. 77 (1972), pp. 9-20.

[4] M. D. Morley, Partitions and Models in Proceedings of the Summer School in Logio Leeds 196\%, Heidelberg 1969.

[5] - and R. L. Vaught, Homogeneous universal models, Math. Scandinovica 11 (1962), pp. 37-57.

[6] R. Phillips, A. Minimal extension which is not conservative, Abstract 701-02-7, Notices A.M.S. 20 (1973), p. A-32.

[7] - Omitting types in arithmetic and conservative Extensions (to appear).

[8] S. Shelah, Stability, the f.c.p. and superstabiity model theorelic properties of formulas in first order theory, Annals of Math. Log. 3 (1971), pp. 271-362.

[9] G. Sacks, Saturated Model Theory, 1972.

MICHIGAN STATE UNIVERSITY

Accepté par la Rédaction le 12.11. 1973 